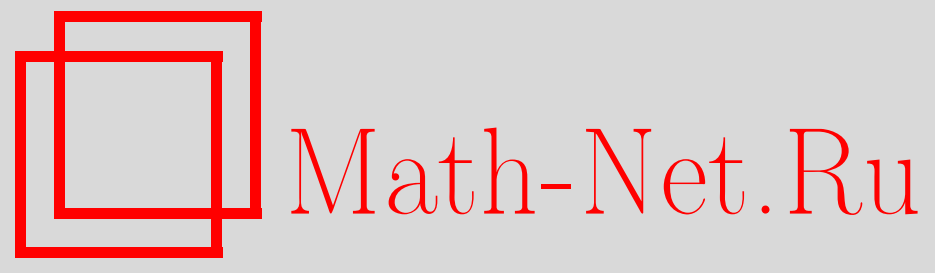

О. А. Репин, С. К. Кумыкова, Внутреннекраевая задача с операторами Римана-Лиувилля для уравнения смешанного типа третьего порядка, Bестн. Сам. гос. техн. ун-та. Сер. Физ.-мат. науки, 2016, номер 1, 43-53

DOI: https://doi.org/10.14498/vsgtu1461

Использование Общероссийского математического портала MathNet.Ru подразумевает, что вы прочитали и согласны с пользовательским соглашением

http://www.mathnet.ru/rus/agreement

Параметры загрузки:

IP : 54.209 .52 .79

26 апреля 2023 г., $15: 36: 34$

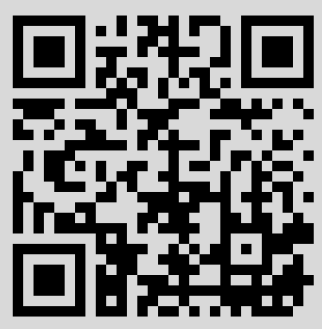


УДК 517.956.6

\title{
ВНУТРЕННЕКРАЕВАЯ ЗАДАЧА С ОПЕРАТОРАМИ РИМАНА-ЛИУВИЛЛЯ ДЛЯ УРАВНЕНИЯ СМЕШАННОГО ТИПА ТРЕТЬЕГО ПОРЯДКА
}

\author{
О. А. Репин ${ }^{1,2}$, C. К. Кумыкова \\ 1 Самарский государственный экономический университет, \\ Россия, 443090, Самара, ул. Советской Армии, 141. \\ 2 Самарский государственный технический университет, \\ Россия, 443100, Самара, ул. Молодогвардейская, 244. \\ 3 Кабардино-Балкарский государственный университет им. Х. М. Бербекова \\ Россия, 360004, Нальчик, ул. Чернышевского, 173.
}

\begin{abstract}
Аннотация
Исследован вопрос однозначной разрешимости внутреннекраевой задачи с операторами Римана-Лиувилля в краевом условии для уравнения смешанного типа третьего порядка. При ограничениях неравенственного типа на известные функции и различных порядках операторов дробного интегро-дифференцирования доказана теорема единственности. Существование решения задачи установлено путем редукции к уравнениям Фредгольма второго рода, безусловная разрешимость которых следует из единственности решения задачи.
\end{abstract}

Ключевые слова: уравнение смешанного типа, уравнение Фредгольма, задача Коши, операторы дробного в смысле Римана-Лиувилля интегродифференцирования.

Теория краевых задач для уравнений смешанного типа является одним из важных разделов современной теории дифференциальных уравнений с частными производными и представляет как теоретический, так и практический интерес. Это обусловлено непосредственными связями уравнений смешанного типа с прикладными задачами околозвуковой газовой динамики, математической биологии, теории бесконечно малых изгибаний поверхностей, магнитной гидродинамики и задачами в других областях.

1. Постановка задачи. Рассмотрим уравнение

$$
0= \begin{cases}u_{x x x}-u_{y}, & y>0 \\ y^{2 m} u_{x x}+y u_{y y}+\alpha u_{y}, & y<0\end{cases}
$$

(C) 2016 Самарский государственный технический университет.

\section{Образец для цитирования}

Р епин О. А., Кумыкова С. К. Внутреннекраевая задача с операторами РиманаЛиувилля для уравнения смешанного типа третьего порядка // Bестн. Сам. гос. техн. ун-та. Сер. Физ.-мат. науки, 2016. Т. 20, № 1. С. 43-53. doi: 10.14498/vsgtu1461.

\section{Сведения об авторах}

Олег Александрович Репин (д.ф.-м.н., проф.; matstat@mail.ru; автор, ведущий переписку), заведующий кафедрой, каф. математической статистики и эконометрики ${ }^{1}$ професcop, каф. прикладной математики и информатики ${ }^{2}$.

Светлана Каншубиевна Кумыкова (к.ф.-м.н., доц.; bsk@rect.kbsu.ru), доцент, каф. математического анализа и теории функций. 
где $m$ - натуральное число, $\alpha=$ const, $(1-2 m) / 2<\alpha<1$ в конечной области $\Omega$, ограниченной отрезками $A A_{0}, B B_{0}, A_{0} B_{0}$ прямых $x=0, x=1, y=1$ соответственно и характеристиками

$$
A C: x-\frac{2}{2 m+1}(-y)^{\frac{2 m+1}{2}}=0, \quad B C: x+\frac{2}{2 m+1}(-y)^{\frac{2 m+1}{2}}=1
$$

уравнения (1) при $y<0$.

Пусть $\Omega_{1}=\Omega \cap(y>0), \Omega_{2}=\Omega \cap(y<0), I \equiv A B-$ единичный интервал $0<x<1$ прямой $y=0$.

ЗАДАчА. Найти функиию $u(x, y) \in C(\bar{\Omega}) \cap C^{3,1}\left(\Omega_{1}\right) \cap C^{2,2}\left(\Omega_{2}\right)$, являющуюся решением уравнения (1) в $\Omega$ при $y \neq 0$ и удовлетворяющую условиям

$$
\begin{gathered}
u(0, y)=\varphi_{1}(y), \quad u(1, y)=\varphi_{2}(y), \quad u_{x}(0, y)=\varphi_{3}(y), \quad 0 \leqslant y \leqslant 1, \\
a(x) D_{0 x}^{\alpha_{1}} \delta(x) u\left[\Theta_{0}(x)\right]+b(x) D_{x 1}^{\beta_{1}} w(x) u\left[\Theta_{1}(x)\right]+c(x) u(x, 0)+ \\
+d(x) \lim _{y \rightarrow-0}(-y)^{\alpha} \frac{\partial u}{\partial y}=\gamma(x), \quad \forall x \in I
\end{gathered}
$$

и условию сопряљения

$$
\lim _{y \rightarrow 0+} u_{y}(x, y)=\lim _{y \rightarrow-0}(-y)^{\alpha} u_{y}(x, y),
$$

где $\varphi_{i}(y)(i=\overline{1,3}), a(x), b(x), c(x), d(x), \gamma(x), \delta(x), w(x)$-непреръьвнье функиии, причем

$$
\begin{gathered}
a^{2}(x)+b^{2}(x)+c^{2}(x)+d^{2}(x) \neq 0 \\
a(x), b(x), c(x), d(x), \gamma(x) \in C^{1}(\bar{I}) \cap C^{3}(I), \quad \varphi_{i}(y) \in C[0,1],
\end{gathered}
$$

$\Theta_{0}(x), \Theta_{1}(x)$ - точки пересечения характеристик уравнения $(1)$, выходящих из точки $(x, 0) \in I$ c характеристиками $A C, B C$ соответственно; $D_{0 x}^{l}$, $D_{x 1}^{l}$ - операторы дробного в смысле Римана-Лиувилля интегро-дифберениирования $[1,2]$.

Задача (1)-(4) относится к классу задач со смещением [3]. Нелокальные задачи со смещением для уравнений смешанного типа ранее исследовались авторами статьи в работах [4, с. 57-65], [5, с. 755-763], [6, с. 17-25]. Данная работа продолжает эти исследования.

\section{2. Единственность решения задачи.}

Теорема. В области $\Omega$ не может существовать более одного решения задачи (1)-(4) при $(1-2 m) / 2<\alpha<1$, если либо

$$
\begin{gathered}
\alpha_{1}=\beta_{1}=1-\beta, \quad w(x)=\delta(x) \equiv 1, \\
M_{1}(x)=\gamma_{1}(1-x)^{\beta} a(x)+\gamma_{1} x^{\beta} b(x)-\frac{\Gamma(\beta)}{\Gamma(2 \beta)} x^{\beta}(1-x)^{\beta} d(x) \neq 0, \\
{\left[\frac{(1-x)^{\beta} a(x)}{M_{1}(x)}\right]^{\prime} \leqslant 0,\left[\frac{x^{\beta} b(x)}{M_{1}(x)}\right]^{\prime} \geqslant 0, \frac{x^{\beta}(1-x)^{\beta} c(x)}{M_{1}(x)} \geqslant 0, \forall x \in \bar{I},}
\end{gathered}
$$

либо

$$
\alpha_{1}=\beta_{1}=\beta, \quad \delta(x)=x^{2 \beta-1}, \quad w(x)=(1-x)^{2 \beta-1}
$$


и выполняются условия

$$
\begin{gathered}
M_{2}(x)=(1-x)^{1-\beta} a(x)+x^{1-\beta} b(x)+\frac{\Gamma(\beta)}{\Gamma(2 \beta)} x^{1-\beta}(1-x)^{1-\beta} c(x) \neq 0, \\
{\left[\frac{\gamma_{1}(1-x)^{\beta} a(x)}{M_{2}(x)}\right]^{\prime} \leqslant 0,\left[\frac{\gamma_{1} x^{1-\beta} b(x)}{M_{2}(x)}\right]^{\prime} \geqslant 0, \frac{x^{1-\beta}(1-x)^{1-\beta} d(x)}{M_{2}(x)} \leqslant 0, \forall x \in \bar{I},}
\end{gathered}
$$

где

$$
\gamma_{1}=\frac{\Gamma(\beta) \Gamma(1-2 \beta)}{2 \Gamma(2 \beta) \Gamma(1-\beta)}\left(\frac{2 m+1}{4}\right)^{-2 \beta}
$$

Доказательство. Регулярное в области $\Omega_{2}$ решение уравнения (1) при $(1-2 m) / 2<\alpha<1$, удовлетворяющее условиям

$$
u(x, 0)=\tau(x), \quad \lim _{y \rightarrow-0}(-y)^{\alpha} u_{y}=\nu(x), \quad 0<x<1,
$$

единственно и имеет вид [7]

$$
\begin{aligned}
& u(x, y)=\frac{\Gamma(2 \beta)}{\Gamma^{2}(\beta)} \int_{0}^{1} \tau\left[x+\frac{2(1-2 t)}{2 m+1}(-y)^{\frac{2 m+1}{2}}\right] t^{\beta-1}(1-t)^{\beta-1} d t- \\
& -\frac{2}{m+1} \frac{\Gamma(1-2 \beta)}{\Gamma^{2}(1-\beta)}(-y)^{1-\alpha} \int_{0}^{1} \nu\left[x+\frac{2(1-2 t)}{2 m+1}(-y)^{\frac{2 m+1}{2}}\right] t^{-\beta}(1-t)^{-\beta} d t
\end{aligned}
$$

где

$$
\beta=\frac{2 m-1+2 \alpha}{2(2 m+1)} .
$$

При выполнении условий (5) теоремы соотношение между $\tau(x)$ и $\nu(x)$ из области $\Omega_{2}$ запишем в виде

$$
\nu(x)=A_{1}(x) D_{0 x}^{1-2 \beta} \tau(x)+B_{1}(x) D_{x 1}^{1-2 \beta} \tau(x)+C_{1}(x) \tau(x)+F_{1}(x),
$$

где

$$
\begin{gathered}
A_{1}(x)=\frac{(1-x)^{\beta} a(x)}{M_{1}(x)}, \quad B_{1}(x)=\frac{x^{\beta} b(x)}{M_{1}(x)}, \\
C_{1}(x)=\frac{x^{\beta}(1-x)^{\beta} c(x)}{M_{1}(x)}, \quad F_{1}(x)=-\frac{\Gamma(\beta)}{\Gamma(2 \beta)} \frac{x^{\beta}(1-x)^{\beta} \gamma(x)}{M_{1}(x)} .
\end{gathered}
$$

Рассмотрим интеграл

$$
I^{*}=\int_{0}^{1} \tau(x) \nu(x) d x
$$

при $\gamma(x)=0$. Подставляя $\nu(x)$, будем иметь

$$
\Gamma(2 \beta) I^{*}=\int_{0}^{1} A_{1}(x) \tau(x)\left[\frac{d}{d x} \int_{0}^{x} \frac{\tau(t) d t}{(x-t)^{1-2 \beta}}\right] d x+\Gamma(2 \beta) \int_{0}^{1} C_{1}(x) \tau^{2}(x) d x-
$$




$$
-\int_{0}^{1} B_{1}(x) \tau(x)\left[\frac{d}{d x} \int_{x}^{1} \frac{\tau(t) d t}{(t-x)^{1-2 \beta}}\right] d x
$$

С учетом обозначений

$$
\frac{\sin (2 \pi \beta)}{\pi} \frac{d}{d x} \int_{0}^{x} \frac{\tau(t) d t}{(x-t)^{1-2 \beta}}=\tau_{1}(x), \quad-\frac{\sin (2 \pi \beta)}{\pi} \frac{d}{d x} \int_{x}^{1} \frac{\tau(t) d t}{(t-x)^{1-2 \beta}}=\tau_{2}(x)
$$

и формулы обращения интегрального уравнения Абеля, получим

$$
\begin{array}{r}
\Gamma(2 \beta) I^{*}=\frac{\pi}{\sin (2 \pi \beta)} \int_{0}^{1} A_{1}(x) \tau_{1}(x) d x \int_{0}^{x} \frac{\tau_{1}(\xi) d \xi}{(x-\xi)^{2 \beta}}+\Gamma(2 \beta) \int_{0}^{1} C_{1}(x) \tau^{2}(x) d x+ \\
+\frac{\pi}{\sin (2 \pi \beta)} \int_{0}^{1} B_{1}(x) \tau_{2}(x) d x \int_{x}^{1} \frac{\tau_{2}(\xi) d \xi}{(\xi-x)^{2 \beta}} .
\end{array}
$$

Воспользуемся известной формулой для гамма-функции [1]

$$
\int_{0}^{\infty} t^{\mu-1} \cos (k t) d t=\frac{\Gamma(\mu)}{k^{\mu}} \cos \left(\frac{\mu \pi}{2}\right), \quad k>0, \quad 0<\mu<1 .
$$

Полагая $k=|x-\xi|, \mu=2 \beta$, получим

$$
\frac{1}{|x-\xi|^{2 \beta}}=\frac{1}{\Gamma(2 \beta) \cos (\pi \beta)} \int_{0}^{\infty} t^{2 \beta-1} \cos (t|x-\xi|) d t
$$

и, следовательно,

$$
\begin{aligned}
\frac{1}{\pi} \Gamma^{2}(2 \beta) \sin (2 \pi \beta) \cos (\pi \beta) I^{*} & = \\
& =\int_{0}^{1} A_{1}(x) \tau_{1}(x) d x \int_{0}^{x} \tau_{1}(\xi) d \xi \int_{0}^{\infty} t^{2 \beta-1} \cos (t|x-\xi|) d t+ \\
& +\int_{0}^{1} B_{1}(x) \tau_{2}(x) d x \int_{x}^{1} \tau_{2}(\xi) d \xi \int_{0}^{\infty} t^{2 \beta-1} \cos (t|\xi-x|) d t+ \\
& +\frac{1}{\pi} \Gamma^{2}(2 \beta) \sin (2 \pi \beta) \cos (\pi \beta) \int_{0}^{1} C_{1}(x) \tau^{2}(x) d x .
\end{aligned}
$$

Поменяв порядок интегрирования, а затем интегрируя по частям с учетом $B_{1}(0)=A_{1}(1)=0$, будем иметь

$$
\frac{1}{\pi} \Gamma^{2}(2 \beta) \sin (2 \pi \beta) \cos (\pi \beta) I^{*}=
$$

$$
=\frac{1}{\pi} \Gamma^{2}(2 \beta) \sin (2 \pi \beta) \cos (\pi \beta) \int_{0}^{1} C_{1}(x) \tau^{2}(x) d x-
$$

$-\frac{1}{2} \int_{0}^{\infty} t^{2 \beta-1} d t \int_{0}^{1} A_{1}^{\prime}(x)\left[\left(\int_{0}^{x} \tau_{1}(\xi) \cos (t \xi) d \xi\right)^{2}+\left(\int_{0}^{x} \tau_{1}(\xi) \sin (t \xi) d \xi\right)^{2}\right] d x+$ $+\frac{1}{2} \int_{0}^{\infty} t^{2 \beta-1} d t \int_{0}^{1} B_{1}^{\prime}(x)\left[\left(\int_{x}^{1} \tau_{2}(\xi) \cos (t \xi) d \xi\right)^{2}+\left(\int_{x}^{1} \tau_{2}(\xi) \sin (t \xi) d \xi\right)^{2}\right] d x$ 
При выполнении условий (8) теоремы соотношение между $\tau(x)$ и $\nu(x)$ из области $\Omega_{2}$ имеет вид

$$
\tau(x)=A_{2}(x) D_{0 x}^{2 \beta-1} \nu(x)+B_{2}(x) D_{x 1}^{2 \beta-1} \nu(x)-C_{2}(x) \nu(x)+F_{2}(x),
$$

где

$$
\begin{gathered}
A_{2}(x)=\frac{\gamma_{1}(1-x)^{1-\beta} a(x)}{M_{2}(x)}, \quad B_{2}(x)=\frac{\gamma_{1} x^{1-\beta} b(x)}{M_{2}(x)}, \\
C_{2}(x)=\frac{x^{1-\beta}(1-x)^{1-\beta} d(x)}{M_{2}(x)}, \quad F_{2}(x)=\frac{\Gamma(\beta)}{\Gamma(2 \beta)} \frac{x^{1-\beta}(1-x)^{1-\beta} \gamma(x)}{M_{2}(x)} .
\end{gathered}
$$

При $\gamma(x)=0$, учитывая $(14)$, а также что $A_{2}(1)=B_{2}(0)=0$, преобразованиями, аналогичными приведенным, получим

$$
\begin{aligned}
& \frac{\pi}{2 \sin (\pi \beta)} I^{*}=-\frac{2}{\pi} \sin (\pi \beta) \int_{0}^{1} C_{2}(x) \nu^{2}(x) d x- \\
& -\frac{1}{2} \int_{0}^{\infty} t^{2 \beta-1} d t \int_{0}^{1} A_{2}^{\prime}(x)\left[\left(\int_{0}^{x} \nu(\xi) \cos (t \xi) d \xi\right)^{2}+\left(\int_{0}^{x} \nu(\xi) \sin (t \xi) d \xi\right)^{2}\right] d x+ \\
& +\frac{1}{2} \int_{0}^{\infty} t^{2 \beta-1} d t \int_{0}^{1} B_{2}^{\prime}(x)\left[\left(\int_{x}^{1} \nu(\xi) \cos (t \xi) d \xi\right)^{2}+\left(\int_{x}^{1} \nu(\xi) \sin (t \xi) d \xi\right)^{2}\right] d x
\end{aligned}
$$

Из (15) и (17) видно, что при выполнении условий (5)-(10) теоремы $I^{*} \geqslant 0$.

$\mathrm{C}$ другой стороны, переходя в уравнении (1) к пределу при $y \rightarrow+0$, получим функциональное соотношение между $\tau(x)$ и $\nu(x)$, принесенное из области $\Omega_{1}$ на линию $y=0$ :

$$
\tau^{\prime \prime \prime}(x)-\nu(x)=0
$$

Подставив $\nu(x)=\tau^{\prime \prime \prime}(x)$ в

$$
I^{*}=\int_{0}^{1} \tau(x) \nu(x) d x
$$

будем иметь

$$
I^{*}=\int_{0}^{1} \tau(x) \tau^{\prime \prime \prime}(x) d x=\int_{0}^{1} \tau(x) d\left[\tau^{\prime \prime}(x)\right] .
$$

Интегрируя по частям с учетом однородных граничных условий (2)

$$
\tau(0)=\tau(1)=\tau^{\prime}(0)=0
$$

нетрудно усмотреть, что

$$
I^{*}=-\frac{\left[\tau^{\prime}(1)\right]^{2}}{2} \leqslant 0 .
$$

Отсюда заключаем, что $I^{*}=0$. Поскольку слагаемые в правых частях (17) и (15) неотрицательны, они также равны нулю. 
В частности, из (17)

$\int_{0}^{\infty} t^{2 \beta-1} d t\left(\int_{0}^{1} \nu(\xi) \cos (t \xi) d \xi\right)^{2}=0, \quad \int_{0}^{\infty} t^{2 \beta-1} d t\left(\int_{0}^{1} \nu(\xi) \sin (t \xi) d \xi\right)^{2}=0$

Интегралы

$$
\int_{0}^{1} \nu(\xi) \cos (t \xi) d \xi=0, \quad \int_{0}^{1} \nu(\xi) \sin (t \xi) d \xi=0
$$

для всех $t \in(0, \infty)$, в частности, при $t=2 \pi k, k=0,1,2, \ldots$, так как $t^{2 \beta-1} \geqslant 0$. При этих значениях $t$ функции $\sin (t \xi)$ и $\cos (t \xi)$ образуют полную ортогональную систему функций в $L^{2}$. Следовательно, $\nu(\xi)=0$ почти всюду, а так как функция $\nu(\xi)$ непрерывна по условию, $\nu(\xi)=0$ всюду. Отсюда и из (16) при $\gamma(x)=0$ имеем $\tau(\xi)=0$.

Аналогичными рассуждениями из (15) можно получить $\tau_{i}(x)=0, i=1,2$ и, следовательно, $\tau(x)=0$, а из (13) при $\gamma(x)=0$ имеем $\nu(x)=0$. Таким образом, решение задачи $u(x, y) \equiv$ в $\Omega_{2}$ как решение задачи (11) с нулевыми данными, а в области $\Omega_{1}$ как решение однородной задачи $(1),(2)$.

3. Существование решения задачи. Интегрируя трижды от 0 до $x$ уравнение $u_{x x x}-u_{y}=0$ и учитывая условия (2), получим

$$
\begin{aligned}
\tau(x)=\frac{1}{2} \int_{0}^{x}(x-\xi)^{2} \nu(\xi) d \xi-\frac{x^{2}}{2} \int_{0}^{1}(1-\xi)^{2} \nu(\xi) d \xi+\varphi_{2}(0) x^{2}+ \\
+\left(x-x^{2}\right) \varphi_{3}(0)+\left(1-x^{2}\right) \varphi_{1}(0) .
\end{aligned}
$$

При выполнении условий $(8)$, исключив $\tau(x)$ из $(16)$ и $(18)$, будем иметь

$$
\begin{aligned}
& \frac{A_{2}(x)}{\Gamma(1-2 \beta)} \int_{0}^{x} \frac{\nu(\xi) d \xi}{(x-\xi)^{2 \beta}}+\frac{B_{2}(x)}{\Gamma(1-2 \beta)} \int_{x}^{1} \frac{\nu(\xi) d \xi}{(\xi+x)^{2 \beta}}-C_{2}(x) \nu(x)+F_{2}(x)= \\
= & \frac{1}{2} \int_{0}^{x}(x-\xi)^{2} \nu(\xi) d \xi-\frac{x^{2}}{2} \int_{0}^{1}(1-\xi)^{2} \nu(\xi) d \xi+\varphi_{2}(0) x^{2}+\left(x-x^{2}\right) \varphi_{3}(0)+\left(1-x^{2}\right) \varphi_{1}(0) .
\end{aligned}
$$

Сгруппировав соответствующие слагаемые, перепишем последнее в виде

$$
C_{2}(x) \nu(x)+\int_{0}^{1} \frac{K(x, \xi) \nu(\xi) d \xi}{|x-\xi|^{2 \beta}}=F_{3}(x)
$$

где

$$
\begin{gathered}
F_{3}(x)=F_{2}(x)-\varphi_{2}(0) x^{2}-\left(x-x^{2}\right) \varphi_{3}(0)-\left(1-x^{2}\right) \varphi_{1}(0), \\
K(x, \xi)= \begin{cases}\frac{1}{2}(x-\xi)^{2+2 \beta}-\frac{A_{2}(x)}{\Gamma(1-2 \beta)}-\frac{x^{2}}{2}(1-\xi)(x-\xi)^{2 \beta} & \text { при } \xi \leqslant x, \\
-\frac{B_{2}(x)}{\Gamma(1-2 \beta)}-\frac{x^{2}}{2}(1-\xi)(\xi-x)^{2 \beta} & \text { при } \xi \geqslant x .\end{cases}
\end{gathered}
$$

Ядро $K(x, \xi) \in C(\bar{I} \times \bar{I}) \cap C^{1}(I \times I)$, правая часть $F_{3}(x) \in C(\bar{I}) \cap C^{3}(I)$. 
Уравнение $(19)$ при $C_{2}(x) \neq 0$ есть уравнение Фредгольма второго рода, безусловная разрешимость которого в требуемом классе функций следует из единственности решения задачи (1)-(4).

При выполнении условий $(5)$ исключим $\nu(x)$ из $(18)$ и $(13)$, получим

$$
\begin{aligned}
\tau(x)=\frac{1}{2 \Gamma(2 \beta)} & \int_{0}^{x}(x-\xi)^{2}\left[A_{1}(\xi) \frac{d}{d \xi} \int_{0}^{\xi} \frac{\tau(t) d t}{(\xi-t)^{1-2 \beta}}-\right. \\
& \left.-B_{1}(\xi) \frac{d}{d \xi} \int_{\xi}^{1} \frac{\tau(t) d t}{(t-\xi)^{1-2 \beta}}+\Gamma(2 \beta) C_{1}(\xi) \tau(\xi)\right] d \xi- \\
& -\frac{x^{2}}{2 \Gamma(2 \beta)} \int_{0}^{1}(1-\xi)^{2}\left[A_{1}(\xi) \frac{d}{d \xi} \int_{0}^{\xi} \frac{\tau(t) d t}{(\xi-t)^{1-2 \beta}}-\right. \\
& \left.-B_{1}(\xi) \frac{d}{d \xi} \int_{\xi}^{1} \frac{\tau(t) d t}{(t-\xi)^{1-2 \beta}}+\Gamma(2 \beta) C_{1}(\xi) \tau(\xi)\right] d \xi+ \\
+\frac{1}{2} \int_{0}^{x}(x-\xi)^{2} F_{1}(\xi) d \xi-\frac{x^{2}}{2} \int_{0}^{1}(1-\xi)^{2} F_{1}(\xi) d \xi+\varphi_{2}(0) x^{2}+ &
\end{aligned}
$$

Интегрируя по частям в двойных интегралах, а затем поменяв порядок интегрирования, из (20) получим интегральное уравнение Фредгольма второго рода

$$
\tau(x)+\int_{0}^{1} K_{1}(x, t) \tau(t) d t=F_{1}^{*}(x)
$$

где

$$
\begin{gathered}
F_{1}^{*}(x)=\frac{1}{2} \int_{0}^{x}(x-\xi)^{2} F_{1}(\xi) d \xi-\frac{x^{2}}{2} \int_{0}^{1}(1-\xi)^{2} F_{1}(\xi) d \xi+ \\
\quad+\varphi_{2}(0) x^{2}+\left(x-x^{2}\right) \varphi_{3}(0)+\left(1-x^{2}\right) \varphi_{1}(0), \\
K_{1}(x, t)=\left\{\begin{array}{l}
K_{11}(x, t) \text { при } t \leqslant x, \\
K_{12}(x, t) \text { при } t \geqslant x,
\end{array}\right. \\
K_{11}(x, t)=\frac{1}{2 \Gamma(2 \beta)}\left[\int_{t}^{x} \frac{N_{1}(\xi) d \xi}{(\xi-t)^{1-2 \beta}}+\int_{0}^{t} \frac{N_{2}(\xi) d \xi}{(\xi-t)^{1-2 \beta}}\right]+ \\
+\frac{x^{2}}{2 \Gamma(2 \beta)}\left[\int_{t}^{x} \frac{N_{1}(\xi) d \xi}{(\xi-t)^{1-2 \beta}}+\int_{x}^{1} \frac{N_{1}(\xi) d \xi}{(\xi-t)^{1-2 \beta}}-\int_{0}^{t} \frac{N_{2}(\xi) d \xi}{(\xi-t)^{1-2 \beta}}\right]+ \\
+\frac{1}{2}(x-\xi)^{2} C_{1}(\xi)-\frac{x^{2}}{2}(1-\xi)^{2} C_{1}(\xi)
\end{gathered}
$$$$
K_{12}(x, t)=-\frac{1}{2 \Gamma(2 \beta)} \int_{0}^{x} \frac{N_{2}(\xi) d \xi}{(\xi-t)^{1-2 \beta}}-\frac{x^{2}}{2}(1-\xi)^{2} C_{1}(\xi)+
$$$$
+\frac{x^{2}}{2 \Gamma(2 \beta)}\left[\int_{\xi}^{1} \frac{N_{1}(\xi) d \xi}{(\xi-t)^{1-2 \beta}}-\int_{0}^{x} \frac{N_{2}(\xi) d \xi}{(\xi-t)^{1-2 \beta}}-\int_{x}^{1} \frac{N_{2}(\xi) d \xi}{(\xi-t)^{1-2 \beta}}\right]
$$ 


$$
\begin{gathered}
N_{1}(\xi)=\left[(1-\xi)^{2} A_{1}(\xi)\right]^{\prime}, \quad N_{2}(\xi)=\left[(1-\xi)^{2} B_{1}(\xi)\right]^{\prime}, \\
K_{11}(x, t), K_{12}(x, t) \in C(\bar{I} \times \bar{I}) \cap C^{3}(I \times I), \quad F_{1}^{*}(x) \in C(\bar{I}) \cap C^{3}(I) .
\end{gathered}
$$

Безусловная разрешимость уравнения (21) следует из единственности решения задачи.

По найденному $\tau(x)$ можно из (13) определить $\nu(x)$ и решение задачи (1)(4) в области $\Omega_{2}$ как решение задачи (11) по формуле (12), а в области $\Omega_{2}-$ как решение задачи $(1),(2), u(x, 0)=\tau(x)$.

При $\alpha=(1-2 m) / 2$ решение задачи (11) имеет вид

$$
\begin{aligned}
u(x, y)=\frac{1}{2} \tau[x- & \left.\frac{2}{2 m+1}(-y)^{\frac{2 m+1}{2}}\right]+\frac{1}{2} \tau\left[x+\frac{2}{2 m+1}(-y)^{\frac{2 m+1}{2}}\right]- \\
& -\frac{2}{2 m+1}(-y)^{\frac{2 m+1}{2}} \int_{0}^{1} \nu\left[x+\frac{2(1-2 t)}{2 m+1}(-y)^{\frac{2 m+1}{2}}\right] d t .
\end{aligned}
$$

При выполнении условий (5) теоремы имеем соотношение

$$
\nu(x)=A_{3}(x) \tau^{\prime}(x)+B_{3}(x) \tau(x)+f(x),
$$

где

$$
\begin{gathered}
A_{3}(x)=\frac{b(x)-a(x)}{M_{3}(x)}, \quad B_{3}(x)=-\frac{2 c(x)}{M_{3}(x)}, \quad f(x)=\frac{2 \gamma(x)}{M_{3}(x)}, \\
M_{3}(x)=2 d(x)-a(x)-b(x) \neq 0 .
\end{gathered}
$$

Рассмотрим

$$
\begin{aligned}
I^{*}=\int_{0}^{1} \tau(x) \nu(x) d x=\int_{0}^{1} A_{3}(x) & \tau^{\prime}(x) \tau(x) d x+\int_{0}^{1} B_{3}(x) \tau^{2}(x) d x= \\
= & \frac{1}{2} \int_{0}^{1} A_{3}(x) d\left[\tau^{2}(x)\right]+\int_{0}^{1} B_{3}(x) \tau^{2}(x) d x .
\end{aligned}
$$

Отсюда, учитывая $\tau(0)=\tau(1)=0$, легко получить

$$
I^{*}=-\frac{1}{2} \int_{0}^{1} A_{3}^{\prime}(x) \tau^{2}(x) d x+\int_{0}^{1} B_{3}(x) \tau^{2}(x) d x .
$$

Условия $A_{3}^{\prime}(x) \leqslant 0, B_{3}(x) \geqslant 0$ обеспечивают знак $I^{*} \geqslant 0$. Так как сверху $I^{*} \leqslant 0$, следует, что $I^{*}=0$ и из последнего равенства $\tau(x)=0$, и из (23) $\nu(x)=0$. Тогда из $(22) u(x, y) \equiv 0$ в $\Omega_{2}$. рода

Исключая $\nu(x)$ из (18) и $(23)$, получим уравнение Фредгольма второго

$$
\tau(x)+\int_{0}^{1} K_{3}(x, \xi) \tau(\xi) d \xi=f_{1}(x)
$$

где

$$
f_{1}(x)=\varphi_{2}(0) x^{2}+\left(x-x^{2}\right) \varphi_{3}(0)+\left(1-x^{2}\right) \varphi_{1}(0)+
$$




$$
\begin{array}{r}
+\frac{1}{2} \int_{0}^{x} f(\xi)(x-\xi)^{2} d \xi-\frac{x^{2}}{2} \int_{0}^{1}(1-\xi)^{2} f(\xi) d \xi, \\
K_{3}(x, \xi)=\left\{\begin{array}{r}
\frac{1}{2}\left[A_{3}^{\prime}(\xi)-B_{3}(\xi)\right]\left[(x-\xi)^{2}-x^{2}(1-\xi)^{2}\right]- \\
-A_{3}(\xi)\left[(x-\xi)-x^{2}(1-\xi)\right] \quad \text { при } \quad \xi \leqslant x, \quad \text { при } \quad \xi \geqslant x, \\
-\frac{x^{2}}{2}(1-\xi)\left[A_{2}^{\prime}(\xi)-B_{2}(\xi)\right]+A_{2}(\xi) x^{2}(1-\xi)
\end{array}\right. \\
K_{3}(x, \xi) \in C^{1}(\bar{I} \times \bar{I}) \cap C^{2}(I \times I), \quad f_{1}(x) \in C^{1}(\bar{I}) \cap C^{3}(I) .
\end{array}
$$

Безусловная разрешимость уравнения (24) следует из единственности решения задачи.

В случае $\alpha=(1-2 m) / 2$ и выполнения условия (8) теоремы единственность и существование решения задачи (1)-(4) установлены аналогично.

\section{ORCID}

Олег Александрович Репин: http://orcid.org/0000-0003-1522-3955

\section{БИБЛИОГРАФИЧЕСКИЙ СПИСОК}

1. Самко С. Г., Килбас А. А., Маричев О. И. Интеграль и производные дробного порядка и некоторые их приложения. Минск: Наука и техника, 1987. 688 с.

2. Нахушев А. М. Дробное исчисление и его применение. М.: Физматлит, 2003. 272 с.

3. Нахушев А. М. Задачи со смещением для уравнений в частных производных. М.: Наука, 2006. 287 c.

4. Репин О. А., Кумыкова С. К. Нелокальная задача для уравнения смешанного типа, порядок которого вырождается вдоль линии изменения типа// Изв. вузов. Матем., 2013. № 8 . С. 57-65.

5. Репин О. А., Кумыкова С. К. Об одной нелокальной задаче для уравнения смешанного типа третьего порядка с кратными характеристиками // Дифференц. уравнения, 2015. T. 51, №6. C. 755-763. doi : 10.1134/S0374064115060072.

6. Репин О. А., Кумыкова С. К. Задача со смещением для уравнения третьего порядка с разрывными коэффициентами // Вестн. Сам. гос. техн. ун-та. Сер. Физ.-мат. наукu, 2012. № 4(29). C. 17-25. doi : 10.14498/vsgtu1123.

7. Бицадзе А. В. Некоторые классы уравнений в частных производных. М.: Наука, 1981. $448 \mathrm{c.}$

8. Кумыкова С. К. Об одной задаче с нелокальными краевыми условиями на характеристиках для уравнения смешанного типа// Дифферени. уравнения, 1974. Т. 10, №1. C. $78-88$.

Поступила в редакцию 21/XI/2015;

в окончательном варианте - $13 / \mathrm{II} / 2016$;

принята в печать $-26 / \mathrm{II} / 2016$. 
Vestn. Samar. Gos. Techn. Un-ta. Ser. Fiz.-mat. nauki

[J. Samara State Tech. Univ., Ser. Phys. \& Math. Sci.], 2016, vol. 20, no. 1, pp. $43-53$

ISSN: 2310-7081 (online), 1991-8615 (print)

doi: http://dx.doi.org/10.14498/vsgtu1461

MSC: 35M12

\title{
AN INTERNAL BOUNDARY VALUE PROBLEM WITH THE RIEMANN-LIOUVILLE OPERATOR FOR THE MIXED TYPE EQUATION OF THE THIRD ORDER
}

\author{
O. A. Repin, S. K. Kumykova \\ 1 Samara State Economic University, \\ 141, Sovetskoy Armii st., Samara, 443090, Russian Federation. \\ 2 Samara State Technical University, \\ 244, Molodogvardeyskaya st., Samara, 443100, Russian Federation. \\ 3 Kabardino-Balkarian State University, \\ 173, Chernyshevskogo st., Nalchik, 360004, Russian Federation.
}

\begin{abstract}
The unique solvability of the internal boundary value problem is investigated for the mixed type equation of the third order with Riemann-Liouville operators in boundary condition. The uniqueness theorem is proved for the different orders of operators of fractional integro-differentiation when the inequality constraints on the known functions exist. The existence of solution is verified by the method of reduction to Fredholm equations of the second kind, which unconditional solvability follows from the uniqueness of the solution of the problem.
\end{abstract}

Keywords: mixed type equation, Fredholm equation, Cauchy problem, fractional operators in the sense of Riemann-Liouville integro-differentiation.

\section{ORCID}

Oleg A. Repin: http://orcid.org/0000-0003-1522-3955

\section{REFERENCES}

1. Samko St. G., Kilbas A. A., Marichev O. I. Fractional integrals and derivatives: theory and applications. New York, NY, Gordon and Breach, 1993, xxxvi+976 pp.

2. Nakhushev A. M. Drobnoe ischislenie $i$ ego primenenie [Fractional calculus and its applications]. Fizmatlit, Moscow, 2003, 272 pp. (In Russian)

(C) 2016 Samara State Technical University.

Please cite this article in press as:

Repin O. A., Kumykova S. K. An internal boundary value problem with the RiemannLiouville operator for the mixed type equation of the third order, Vestn. Samar. Gos. Tekhn. Univ., Ser. Fiz.-Mat. Nauki [J. Samara State Tech. Univ., Ser. Phys. \& Math. Sci.], 2016, vol. 20, no. 1, pp. 43-53. doi: 10.14498/vsgtu1461. (In Russian)

\section{Authors Details:}

Oleg A. Repin (Dr. Phys. \& Math. Sci., Professor; matstat@mail.ru; Corresponding Author), Head of Department, Dept. of Mathematical Statistics and Econometrics ${ }^{1}$; Professor, Dept. of Applied Mathematics \& Computer Science ${ }^{2}$.

Svetlana K. Kumykova (Cand. Phys. \& Math. Sci.; bsk@rect.kbsu.ru), Associate Professor, Dept. of Mathematical Analysis and Theory of Functions. 
3. Nakhushev A. M. Zadachi so smeshcheniem dlia uravnenii v chastnykh proizvodnykh [Problems with shifts for partial differential equations]. Moscow, Nauka, 2006, 287 pp. (In Russian)

4. Repin O. A., Kumykova S. K. A nonlocal problem for a mixed-type equation whose order degenerates along the line of change of type, Russian Math. (Iz. VUZ), 2013, vol.57, no. 8, pp. 49-56. doi: 10.3103/S1066369X13080069.

5. Repin O. A., Kumykova S. K. On a nonlocal problem for a third-order equation of mixed type with multiple characteristics, Differ. Equ., 2015, vol.51, no.6, pp. 767-775. doi: 10 . 1134/S0012266115060075.

6. Repin O. A., Kumykova S. K. Problem with shift for the third-order equation with discontinuous coefficients, Vestn. Samar. Gos. Tekhn. Univ. Ser. Fiz.-Mat. Nauki [J. Samara State Tech. Univ., Ser. Phys. \& Math. Sci.], 2012, no.4(29), pp. 17-25 (In Russian). doi : 10.14498/vsgtu1123.

7. Bitsadze A. V. Some classes of partial differential equations, Advanced Studies in Contemporary Mathematics, vol.4. New York, Gordon \& Breach Science Publ., 1988, $\mathrm{xi}+504 \mathrm{pp}$.

8. Kumykova S. K. A problem for an equation of mixed type with nonlocal boundary conditions on characteristics, Differ. Equ., 1974, vol. 10, no. 1, pp. 55-62.

Received 21/XI/2015;

received in revised form $13 / \mathrm{II} / 2016$;

accepted 26/II/2016. 